\title{
The HMG-CoA reductase inhibitor rosuvastatin and the angiotensin receptor antagonist candesartan attenuate atherosclerosis in an apolipoprotein E-deficient mouse model of diabetes via effects on advanced glycation, oxidative stress and inflammation
}

\author{
A. C. Calkin • S. Giunti • K. J. Sheehy $\cdot$ C. Chew • \\ V. Boolell • Y. S. Rajaram • M. E. Cooper • \\ K. A. Jandeleit-Dahm
}

Received: 8 January 2008 / Accepted: 22 April 2008 / Published online: 2 July 2008

(C) Springer-Verlag 2008

\begin{abstract}
Aims/hypothesis We evaluated the anti-atherosclerotic effect of the 3-hydroxy-3-methylglutaryl CoA reductase inhibitor, rosuvastatin, and the angiotensin II receptor blocker (ARB), candesartan, alone and in combination, in the streptozotocin-induced diabetic apolipoprotein Edeficient $\left(\right.$ Apoe $\left.^{-/-}\right)$mouse.

Methods Control and streptozotocin-induced diabetic Apoe ${ }^{-/-}$mice received rosuvastatin $\left(5 \mathrm{mg} \mathrm{kg}^{-1} \mathrm{day}^{-1}\right)$, candesartan $\left(2.5 \mathrm{mg} \mathrm{kg}^{-1} \mathrm{day}^{-1}\right)$, dual therapy or no treatment for 20 weeks. Aortic plaque deposition was assessed by Sudan IV staining and subsequent visual quantification. The abundance of proteins was measured using immunohistochemistry.

Results Diabetes was associated with a fourfold increase in total plaque area. Rosuvastatin attenuated plaque area in diabetic mice in the absence of lipid-lowering effects. The anti-atherosclerotic effect of rosuvastatin was comparable to that observed with candesartan. A similar beneficial effect was seen with dual therapy, although it was not superior to
\end{abstract}

Electronic supplementary material The online version of this article (doi:10.1007/s00125-008-1060-6) contains supplementary material, which is available to authorised users.

A. C. Calkin $\cdot$ S. Giunti $\cdot$ K. J. Sheehy $\cdot$ C. Chew $\cdot$ V. Boolell $\cdot$

Y. S. Rajaram • M. E. Cooper · K. A. Jandeleit-Dahm $(\square)$

Diabetes Complications Laboratory,

Baker Heart Research Institute,

P.O. Box 6492, St Kilda Rd Central,

Melbourne, 8008 Victoria, Australia

e-mail: karin.jandeleit-dahm@baker.edu.au monotherapy. Rosuvastatin treatment was associated with attenuated accumulation of AGE and AGE receptor (RAGE) in plaques. Similar beneficial effects on markers of oxidative stress were seen with the ARB and statin. Candesartan was more effective at reducing macrophage accumulation and collagen I abundance in plaques compared with rosuvastatin. The combined effect of candesartan and rosuvastatin was superior in reducing macrophage infiltration, monocyte chemoattractant protein-1 level, vascular AGE accumulation and RAGE abundance in the vascular wall. Furthermore, the combination tended to be more effective in reducing smooth muscle cell infiltration and connective tissue growth factor abundance in plaques. Conclusions/interpretation Rosuvastatin has direct antiatherosclerotic effects in diabetic macrovascular disease. These effects are independent of effects on lipids and comparable to the effects observed with candesartan.

Keywords Angiotensin II · Atherosclerosis · Diabetes · Diabetic-hypercholesterolaemic mouse $\cdot$ Statin

$\begin{array}{ll}\text { Abbreviations } \\ \text { ARB } & \text { angiotensin II receptor blocker } \\ \text { AT1 } & \text { angiotensin subtype 1 receptor } \\ \text { CML } & \text { carboxymethyllysine } \\ \text { CTGF } & \text { connective tissue growth factor } \\ \text { HMG-CoA } & \text { 3-hydroxy-3-methylglutaryl CoA } \\ \text { MCP-1 } & \text { monocyte chemoattractant protein-1 } \\ \text { RAC-1 } & \text { ras-related C3 botulinum toxin substrate 1 } \\ \text { RAGE } & \text { AGE receptor }\end{array}$

Abbreviations

ARB angiotensin II receptor blocker

AT1 angiotensin subtype 1 receptor

CML carboxymethyllysine

CTGF connective tissue growth factor

HMG-CoA 3-hydroxy-3-methylglutaryl CoA

MCP-1 monocyte chemoattractant protein-1

RAGE AGE receptor 


\section{Introduction}

It is well established that people with diabetes have a greater risk of heart disease than those without diabetes. Indeed, macrovascular complications account for more than $50 \%$ of deaths in people with diabetes. While it is understood that both metabolic and haemodynamic factors such as hyperglycaemia, hyperlipidaemia and dysregulation of the renin-angiotensin system contribute to diabetesassociated atherosclerosis [1-3], other less traditional pathways, such as oxidative stress and advanced glycation, are now considered to play an important role in the development of diabetic macrovascular disease [4, 5].

Type 2 diabetes is often associated with a range of concomitant disorders including hypertension and dyslipidaemia. 3-Hydroxy-3-methylglutaryl CoA (HMG-CoA) reductase inhibitors, also known as statins, are commonly used for the treatment of dyslipidaemia. A number of large studies have demonstrated that statin therapy is associated with a reduction in cardiovascular events in people both with and without type 2 diabetes [6-12]. While a reduction in LDL-cholesterol was observed in many of these studies, it is now considered that statins also mediate pleiotropic antiatherogenic effects that are independent of their effects on lipoproteins, and that this action may contribute to their efficacy in reducing cardiovascular events. Indeed, HMGCoA reductase inhibitors have been shown to attenuate, although not in the context of diabetes, many of the stages critical to atherosclerotic plaque development including monocyte chemotaxis [13], neutrophil-endothelial cell interaction [13], smooth muscle cell apoptosis [14], migration [15] and proliferation [16], as well as plaque stability [17].

Angiotensin II receptor blockers (ARBs) antagonise the angiotensin subtype 1 receptor (AT1) and are widely used as blood pressure-lowering agents in the absence and presence of diabetes. The prevalence of hypertension is greater in patients with diabetes [18], and hypertension itself is an independent cardiovascular risk factor [19]. A reduction in cardiovascular events has been seen in a number of large studies on agents that interrupt the reninangiotensin system in patients without and with diabetes [20-22], including ARBs [23]. The cardioprotective effects seen with these agents may be due not only to blood pressure reduction, but also to additional mechanisms resulting from a reduction of angiotensin II's direct actions on the vasculature. It is now well established that angiotensin II mediates various direct, pro-atherogenic effects on the vasculature, including the upregulation of monocyte migration [24], endothelial cell adhesion [25], and smooth muscle cell proliferation and migration [26, 27], as well as increased reactive oxygen species [28], proinflammatory cytokines [29] and growth factors [30]. Moreover, various components of the renin-angiotensin system have been shown to be present at the level of the vessel wall $[2,3,30]$.

The aim of the current study was initially to assess such anti-atherosclerotic effects of the HMG-CoA reductase inhibitor rosuvastatin that are independent of its lipidlowering effects, in a model of atherosclerosis accelerated by diabetes, specifically in the streptozotocin-induced diabetic, hyperlipidaemic apolipoprotein E-deficient $\left(\right.$ Apo $\left.^{-/-}\right)$mouse. These effects were then compared with those seen in this model after treatment with the ARB candesartan. Finally, the anti-atherosclerotic effects of combined rosuvastatin and candesartan treatment were assessed and compared with rosuvastatin and candesartan alone, with concomitant evaluation of potentially relevant pathways.

The diabetic Apoe $e^{-/-}$mouse model is associated with accelerated atherosclerosis exhibiting lesions similar to those seen in humans, ranging from fatty streaks to complex plaques with necrotic cores and cholesterol clefts $[2,3]$. Indeed, our laboratory has shown that the diabetic Apoe $e^{-/-}$mouse is an excellent model for studying the effects of various agents on diabetes-associated atherosclerosis, particularly agents that influence the haemodynamic and metabolic pathways that are activated in the diabetic state $[31,32]$.

\section{Methods}

Animals

We used 6-week-old male homozygous Apoe ${ }^{-/-}$mice (back-crossed twenty times from the C57BL/6 strain; Animal Resource Centre, Canning Vale, WA, Australia), which were housed at the Precinct Animal Centre at the Alfred Monash Research Education Precinct (Melbourne, Australia) and studied according to the principles devised by the Animal Welfare Committee of the Baker Heart Research Institute and Alfred Hospital. A subset of mice was rendered diabetic by five daily intraperitoneal injections of streptozotocin (Boehringer-Mannheim, Mannheim, Germany), at a dose of $55 \mathrm{mg} / \mathrm{kg}$ in citrate buffer [2]. Control (buffer alone) and diabetic mice were further randomised to receive: (1) the $\mathrm{HMG}-\mathrm{CoA}$ reductase inhibitor rosuvastatin (5 $\mathrm{mg} \mathrm{kg}^{-1}$ day $^{-1}$; AstraZeneca, Macclesfield, UK); (2) the AT1 receptor antagonist candesartan (2.5 $\mathrm{mg} \mathrm{kg}^{-1}$ day $^{-1}$; AstraZeneca); (3) dual therapy by gavage at the above-mentioned doses for 20 weeks; or (4) no treatment ( $n=6-8$ per group).

Systolic blood pressure was assessed by a non-invasive tail cuff system in conscious mice at the end of the study [33]. At the conclusion of the study, animals were anaesthetised by an intraperitoneal injection of pentobarbitone 
sodium/phenytoin sodium (100 $\mathrm{mg} / \mathrm{kg}$ body weight) (Euthatal; Sigma-Aldrich, Castle Hill, NSW, Australia). Blood was collected from the left ventricle and centrifuged $(6,000 \times g)$, and plasma and erythrocytes were stored at $-20^{\circ} \mathrm{C}$ and $4^{\circ} \mathrm{C}$ respectively for subsequent analysis. Animals were killed and the aortas were rapidly dissected and snap frozen in liquid nitrogen and stored at $-80^{\circ} \mathrm{C}$ or stored in buffered formalin $(10 \%$, vol./vol.) for subsequent measurement of plaque area and immunohistochemical studies.

\section{Metabolic parameters}

Glycohaemoglobin was determined in lysates of erythrocytes by high-pressure liquid chromatography (BioRad, Richmond, CA, USA) [34]. Plasma cholesterol, triacylglycerol and glucose levels were measured by an autoanalyser technique (Hitachi 917; Hitachi, Tokyo, Japan); LDLcholesterol was calculated according to the Friedewald formula [35].

\section{Assessment of plaque area}

To evaluate the atherosclerotic lesions, two approaches were used: visual analysis and histological section analysis. The visual approach was used to obtain information about the distribution and extent of atherosclerosis throughout the aorta, whereas microscopic analysis was used to evaluate lesion composition. In brief, as previously described, the aorta was cleaned of peripheral fat under a dissecting microscope and stained with Sudan IV-Herxheimer's solution ( $0.5 \%$ wt/vol.; Sigma Chemical, St Louis, MO, USA) [2]. Aortic images were digitised using a dissecting microscope (Olympus SZX9; Olympus Optical, Tokyo, Japan) equipped with a high-resolution camera (Zeiss, Heidelberg, Germany). Lesion area measurements were performed by calculating the proportion of aortic intima surface area occupied by the red stain in each of the segments.
Immunohistochemistry

Serial sections were dewaxed and rehydrated as described previously [2]. For detailed information, please see Electronic supplementary material (ESM; Methods and ESM Table 1).

\section{Statistical analysis}

Data were analysed by ANOVA using Statview V (Brainpower, Calabasas, CA, USA). Post hoc comparisons of group means were performed by Fisher's least significant difference method. Data are shown as mean \pm SEM unless otherwise specified. A $p$ value of less than 0.05 was regarded as statistically significant.

\section{Results}

Metabolic parameters and blood pressure

Diabetes was associated with reduced body weight gain in streptozotocin-treated Apoe ${ }^{--}$mice compared with nondiabetic control Apoe $e^{-/-}$mice (Table 1). Body weights were not influenced by any of the treatments, either as monotherapies or in combination (Table 1). Food intake was increased in diabetic animals, but was not affected by any of the treatments (Table 1).

Diabetes was associated with a marked elevation in glycated haemoglobin and plasma glucose levels (Table 1). Neither candesartan nor rosuvastatin nor dual therapy had any effect on these parameters.

Diabetes was associated with a marked increase in plasma total cholesterol levels and LDL-cholesterol levels $(p<0.05)$. Neither candesartan nor rosuvastatin nor dual therapy had an effect on any of these parameters (Table 1). Triacylglycerol and HDL concentrations were increased in

Table 1 Characteristics of mice at the conclusion of the 20 week study

\begin{tabular}{|c|c|c|c|c|c|}
\hline Parameters & Control & Diabetic & $\mathrm{D}+\mathrm{Ca}$ & $\mathrm{D}+\mathrm{RSV}$ & $\mathrm{D}+\mathrm{Ca} / \mathrm{RSV}$ \\
\hline Body weight (g) & $30 \pm 0$ & $24 \pm 0 *$ & $24 \pm 1$ & $23 \pm 1$ & $25 \pm 1$ \\
\hline Food intake $(\mathrm{g})$ & $2.1 \pm 0.2$ & $7.0 \pm 0.4^{*}$ & $6.4 \pm 0.3$ & $6.5 \pm 0.2$ & $6.4 \pm 0.3$ \\
\hline Glycated haemoglobin (\%) & $5.3 \pm 0.2$ & $14.8 \pm 0.5^{*}$ & $14.8 \pm 0.5$ & $14.8 \pm 0.8$ & $14.8 \pm 0.8$ \\
\hline Glucose $(\mathrm{mmol} / \mathrm{l})$ & $9.7 \pm 0.4$ & $29.0 \pm 1.4^{*}$ & $29.4 \pm 1.2$ & $27.2 \pm 1.2$ & $28.2 \pm 1.0$ \\
\hline Total cholesterol $(\mathrm{mmol} / \mathrm{l})$ & $11.6 \pm 0.5$ & $24.9 \pm 2.4^{*}$ & $23.3 \pm 1.6$ & $26.8 \pm 1.9$ & $27.4 \pm 2.6$ \\
\hline HDL-cholesterol (mmol/l) & $2.7 \pm 0.2$ & $3.8 \pm 0.4^{*}$ & $4.3 \pm 0.4$ & $4.1 \pm 0.5$ & $4.0 \pm 0.6$ \\
\hline LDL-cholesterol (mmol/l) & $8.3 \pm 0.4$ & $19.5 \pm 2.1^{*}$ & $18.0 \pm 1.1$ & $21.2 \pm 1.4$ & $22.0 \pm 2.1$ \\
\hline Triacylglycerol (mmol/l) & $1.3 \pm 0.2$ & $3.9 \pm 0.8^{*}$ & $2.2 \pm 0.4$ & $3.4 \pm 0.6$ & $3.0 \pm 0.4$ \\
\hline Systolic BP (mmHg) & $100 \pm 4$ & $108 \pm 2 *$ & $96 \pm 4^{\dagger}$ & $107 \pm 3$ & $100 \pm 4$ \\
\hline
\end{tabular}

Data are expressed as mean \pm SEM

$\mathrm{D}+\mathrm{Ca}$, diabetic + candesartan; $\mathrm{D}+\mathrm{RSV}$, diabetic + rosuvastatin; $\mathrm{D}+\mathrm{Ca} / \mathrm{RSV}$, diabetic + candesartan + rosuvastatin

${ }^{*} p<0.05$ vs control group; ${ }^{\dagger} p<0.05$ vs diabetic group; $n=6-8$ per group 

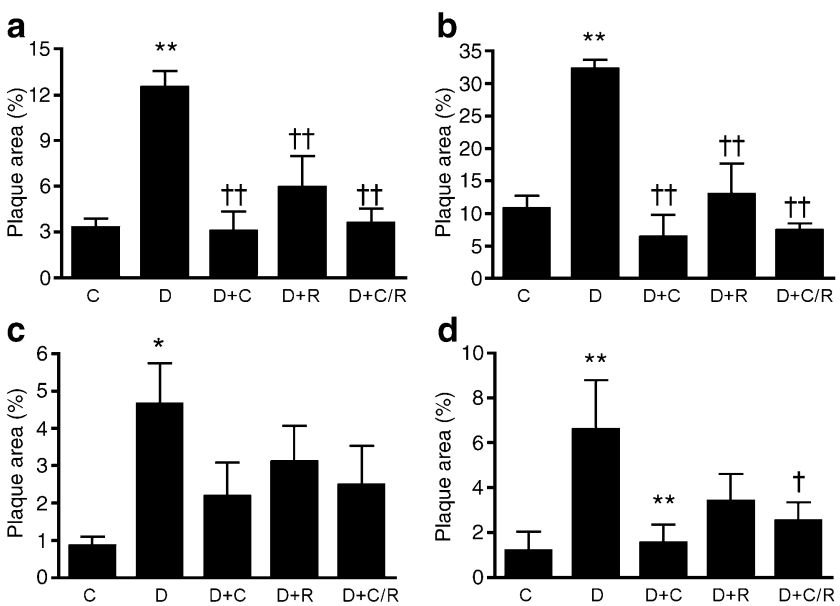

Fig. 1 Quantification of visual plaque area for a total aorta, $\mathbf{b}$ arch, $\mathbf{c}$ thoracic and $\mathbf{d}$ abdominal aorta. Data are expressed as mean \pm SEM. ${ }^{*} p<0.05,{ }^{*} p<0.01$ vs control; ${ }^{\dagger} p<0.05,{ }^{\dagger \dagger} p<0.01$ vs diabetic. C, control; D, diabetic; D + C, diabetic + candesartan; D + R, diabetic + rosuvastatin; $\mathrm{D}+\mathrm{C} / \mathrm{R}$, diabetic + candesartan + rosuvastatin

diabetic Apoe $^{-/-}$mice compared with non-diabetic controls (Table 1), but neither treatment significantly reduced these parameters.

The induction of diabetes was associated with a small but significant increase in systolic blood pressure in $A p o e^{-/-}$ mice (Table 1). Candesartan had a modest blood pressurereducing effect, whereas rosuvastatin and the combination had no effect on blood pressure.

\section{Plaque area}

Diabetes was associated with a fourfold increase in total plaque area $(p<0.01$; Fig. 1a). This increase in plaque area was observed across the whole aorta (arch, thoracic and abdominal segments; $p<0.05$; Fig. 1b-d), but was most apparent in the aortic arch. Both rosuvastatin $(p<0.01)$ and candesartan $(p<0.01)$ were associated with a reduction in total plaque area to levels near those seen in control mice. While dual therapy was also effective at normalising total plaque area $(p<0.01)$, it was not superior to either agent alone. A similar profile of plaque deposition was seen in the aortic arch (Fig. 1b), with all three treatment regimens effective at attenuating atherosclerosis $(p<0.01)$. While, in the thoracic aorta, there was a tendency for all three treatments to attenuate the increase in plaque deposition seen in diabetic mice, none of these reached significance. In the abdominal aorta, candesartan $(p<0.01)$ as well as dual therapy $(p<0.05)$ attenuated atherosclerosis.

Plaque and vessel wall composition

Inflammation Macrophage accumulation, as assessed by F4/80 staining, tended to be increased by $25 \%$ in the plaques of diabetic mice, but this did not reach statistical significance (Table 2). Both candesartan and the combination of candesartan and rosuvastatin attenuated macrophage infiltration $(p<0.05)$ to levels below that seen in control Apoe $^{-/-}$mice. In the adjacent vascular wall there was no difference in F4/80 abundance in diabetic mice (Table 2). Dual therapy was associated with a greater than $50 \%$ reduction in macrophage infiltration $(p<0.05)$, an effect not seen with either candesartan or rosuvastatin alone $(p<0.01$ for candesartan vs combination treatment). Monocyte chemoattractant protein-1 (MCP-1) production was significantly increased in the vascular wall and plaques of diabetic Apoe $^{-/}$mice (Table 2). Candesartan and rosuvastatin alone had only moderate effects on MCP-1 abundance, but the combination of both treatments significantly reduced these in the vascular wall $(p<0.05$ versus diabetes,

Table 2 Quantification of plaque and vessel wall composition

\begin{tabular}{|c|c|c|c|c|c|}
\hline & Control & Diabetic & $\mathrm{D}+\mathrm{Ca}$ & $\mathrm{D}+\mathrm{RSV}$ & $\mathrm{D}+\mathrm{Ca} / \mathrm{RSV}$ \\
\hline \multicolumn{6}{|l|}{$\mathrm{F} 4 / 80$} \\
\hline Plaque & $11.6 \pm 6.2$ & $16.1 \pm 5.5$ & $3.3 \pm 1.4^{\dagger}$ & $7.6 \pm 3.1$ & $2.7 \pm 2.3^{\dagger}$ \\
\hline Vessel wall & $9.0 \pm 0.9$ & $6.8 \pm 1.9$ & $7.8 \pm 1.5$ & $4.5 \pm 1.0$ & $2.5 \pm 1.0^{\dagger}$ \\
\hline \multicolumn{6}{|l|}{ MCP-1 } \\
\hline Plaque & $31.2 \pm 0.4$ & $52.2 \pm 4.6^{* *}$ & $33.6 \pm 0.5^{\dagger \dagger}$ & $47.9 \pm 1.6$ & $36.6 \pm 0.5^{\dagger \dagger}$ \\
\hline Vessel wall & $20.0 \pm 3.3$ & $33.1 \pm 3.1^{* *}$ & $26.7 \pm 2.2$ & $33.0 \pm 2.7$ & $25.1 \pm 1.0^{\dagger}$ \\
\hline \multicolumn{6}{|l|}{ Collagen I } \\
\hline Plaque & $6.3 \pm 4.1$ & $12.9 \pm 5.9$ & $3.4 \pm 1.9^{\dagger}$ & $3.6 \pm 2.2$ & $5.8 \pm 3.3$ \\
\hline Vessel wall & $4.9 \pm 0.7$ & $16.3 \pm 0.8^{*}$ & $12.0 \pm 3.4$ & $8.0 \pm 2.6^{\dagger}$ & $11.0 \pm 1.2$ \\
\hline \multicolumn{6}{|l|}{ Collagen IV } \\
\hline Plaque & $1.5 \pm 1.0$ & $4.9 \pm 2.7$ & $3.8 \pm 1.9$ & $3.8 \pm 1.2$ & $1.8 \pm 1.2$ \\
\hline Vessel wall & $9.7 \pm 2.1$ & $17.1 \pm 1.7^{* *}$ & $8.5 \pm 1.7^{\dagger \dagger}$ & $7.7 \pm 0.8^{\dagger \dagger}$ & $8.8 \pm 1.0^{\dagger \dagger}$ \\
\hline
\end{tabular}

Data are expressed as mean \pm SEM

$\mathrm{D}+\mathrm{Ca}$, diabetic + candesartan; $\mathrm{D}+\mathrm{RSV}$, diabetic + rosuvastatin $; \mathrm{D}+\mathrm{Ca} / \mathrm{RSV}$, diabetic + candesartan + rosuvastatin

${ }^{*} p<0.05,{ }^{* *} p<0.01$ vs control group; ${ }^{\dagger} p<0.05,{ }^{\dagger} p<0.01$ vs diabetic group; $n=6-8$ per group 
$p<0.05$ rosuvastatin versus combination treatment). In the plaque, candesartan and the combination therapy significantly reduced MCP-1 abundance ( $p<0.01$; Table 2).

$A G E / A G E$ receptor axis Diabetes was associated with an approximately fivefold increase in the presence of the AGE, carboxymethyllysine (CML), in plaques of $\mathrm{Apoe}^{-/-}$mice (Fig. 2a,d). Rosuvastatin attenuated AGE accumulation by approximately $50 \%$ in plaques $(p<0.05$; Fig. $2 \mathrm{f}$ ), while candesartan reduced CML abundance to those seen in control mice $(p<0.01$; Fig. 2e). The combination of rosuvastatin and candesartan was associated with a further decrease in vascular CML accumulation $(p<0.01$; Fig. $2 \mathrm{~g})$. Similarly, diabetes induced a sixfold increase in CML deposition in the vascular wall, this being significantly attenuated only in the combination treatment group $(p<0.01$ versus diabetes and candesartan, $p<0.05$ versus rosuvastatin; Fig. 2b).
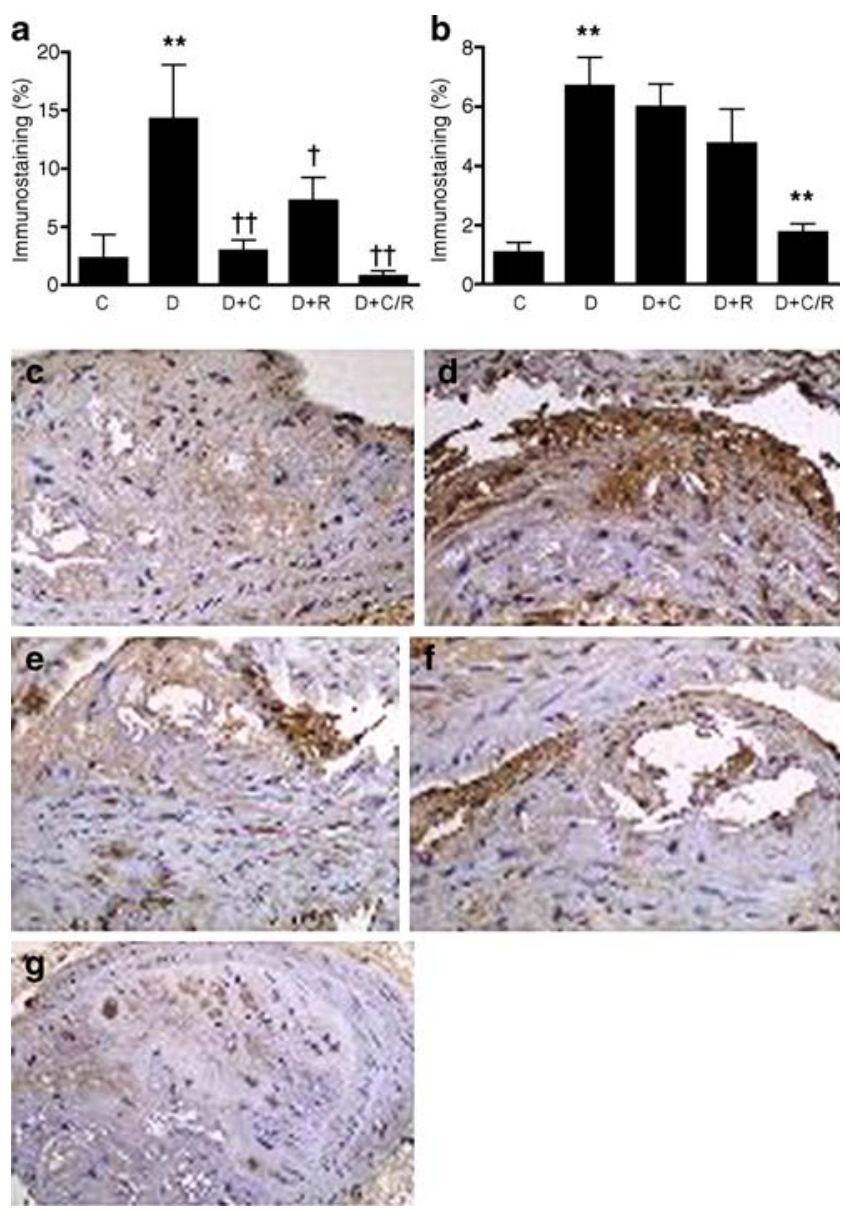

Fig. 2 Accumulation of the AGE CML in the plaque (a) and vessel wall (b). Representative images of vessels of control (c), diabetic (d), diabetic + candesartan (e), diabetic + rosuvastatin (f) and diabetic + candesartan/rosuvastatin $(\mathbf{g})$ mice. Data are expressed as mean $\pm \mathrm{SEM}$. ${ }^{* *} p<0.01$ vs control; ${ }^{\dagger} p<0.05,{ }^{\dagger \dagger} p<0.01$ vs diabetic. Magnification $\times 430$. C, control; $\mathrm{D}$, diabetic; $\mathrm{D}+\mathrm{C}$, diabetic + candesartan; $\mathrm{D}+\mathrm{R}$, diabetic + rosuvastatin; $\mathrm{D}+\mathrm{C} / \mathrm{R}$, diabetic + candesartan + rosuvastatin
The changes in abundance of the AGE receptor (RAGE) were similar to those seen for CML in plaques. Indeed, all three treatments were effective at attenuating the diabetesassociated upregulation of RAGE in the plaques $(p<0.01$; Fig. $3 \mathrm{a}, \mathrm{c}-\mathrm{g}$ ). In the vessel wall, however, RAGE protein tended to be decreased in the rosuvastatin group, with a further decrease in the combination group. These changes, however, did not reach statistical significance (Fig. 3b).

Oxidative stress Nitrotyrosine, a downstream product of nitric oxide and superoxide, is considered to reflect vascular oxidative stress; it was markedly upregulated in the plaques of diabetic mice $(p<0.01$; Fig. 4). Moreover, all three treatments decreased staining of this molecule $(p<0.01)$. A similar pattern was seen in the adjacent vascular wall with all three treatments being effective at attenuating the diabetes-associated upregulation of nitrotyrosine and the combination treatment being the most effective $(p<0.01$ versus rosuvastatin).
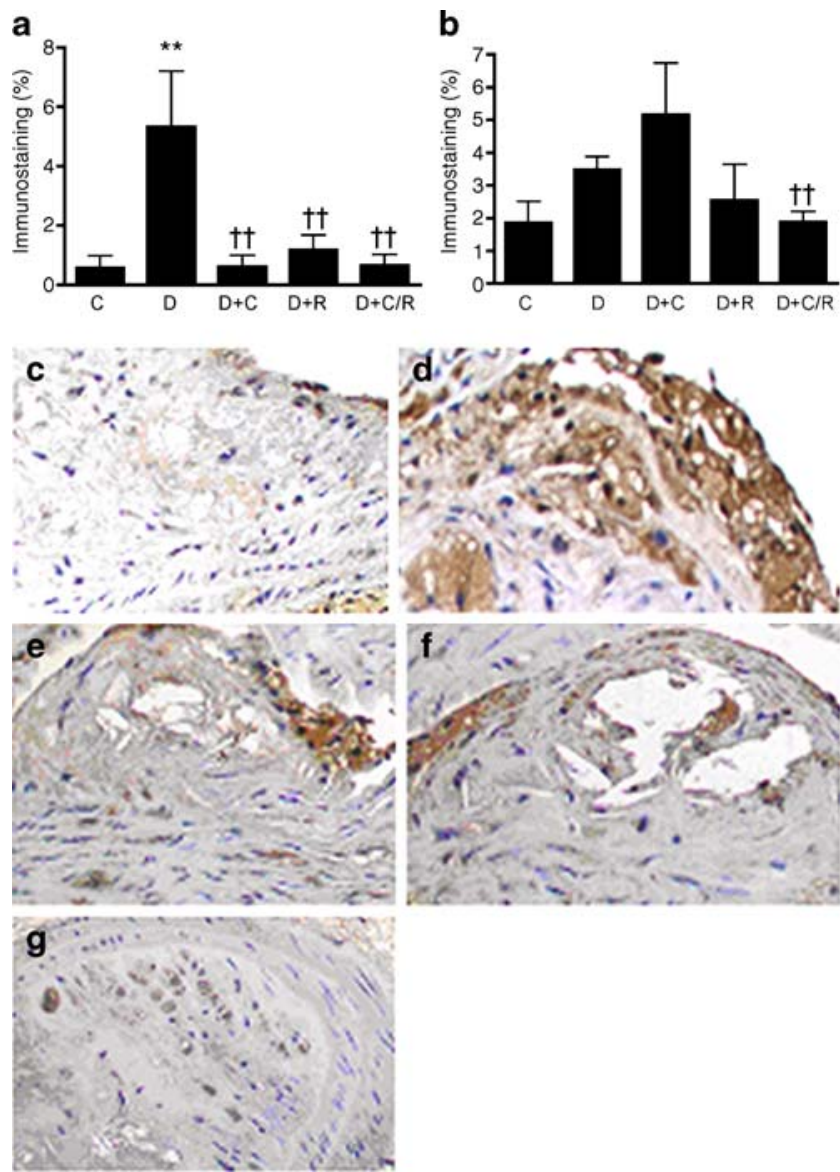

Fig. 3 Immunostaining of RAGE in the plaque (a) and vessel wall (b). Representative images of vessels of control (c), diabetic (d), diabetic + candesartan (e), diabetic + rosuvastatin (f) and diabetic + candesartan/rosuvastatin (g) mice. Data are expressed as mean \pm SEM. ${ }^{* *} p<0.01$ vs control; ${ }^{\dagger \dagger} p<0.01$ vs diabetic. Magnification $\times 430$. C, control; $\mathrm{D}$, diabetic; $\mathrm{D}+\mathrm{C}$, diabetic + candesartan; $\mathrm{D}+\mathrm{R}$, diabetic + rosuvastatin; $\mathrm{D}+\mathrm{C} / \mathrm{R}$, diabetic + candesartan + rosuvastatin 
Diabetes was associated with increased production of the NAD(P)H subunit, p47phox, in plaques of $A p o e^{-/-}$mice $(p<0.05)$, an increase attenuated by rosuvastatin $(p<0.05$; Fig. 5a) and candesartan $(p<0.01)$. As seen in the plaque, there was a marked increase in $\mathrm{p} 47$ phox expression in the vascular wall, which was normalised by all three treatments $(p<0.01$; Fig. 5 b) .

Abundance of the NAD $(\mathrm{P}) \mathrm{H}$ oxidase subunit, gp91phox, in plaques of $A p o e^{-/-}$tended to be increased by diabetes, but none of the treatments studied significantly reduced this parameter (ESM Fig. 1). In the adjacent vessel wall, however, diabetes was associated with an upregulation of gp91phox, which was attenuated by rosuvastatin $(p<0.01)$ and candesartan $(p<0.05)$; no significant effect was seen for combination therapy.

Ras-related C3 botulinum toxin substrate 1 (RAC-1), which is also a subunit of $\mathrm{NAD}(\mathrm{P}) \mathrm{H}$ oxidase, tended to be
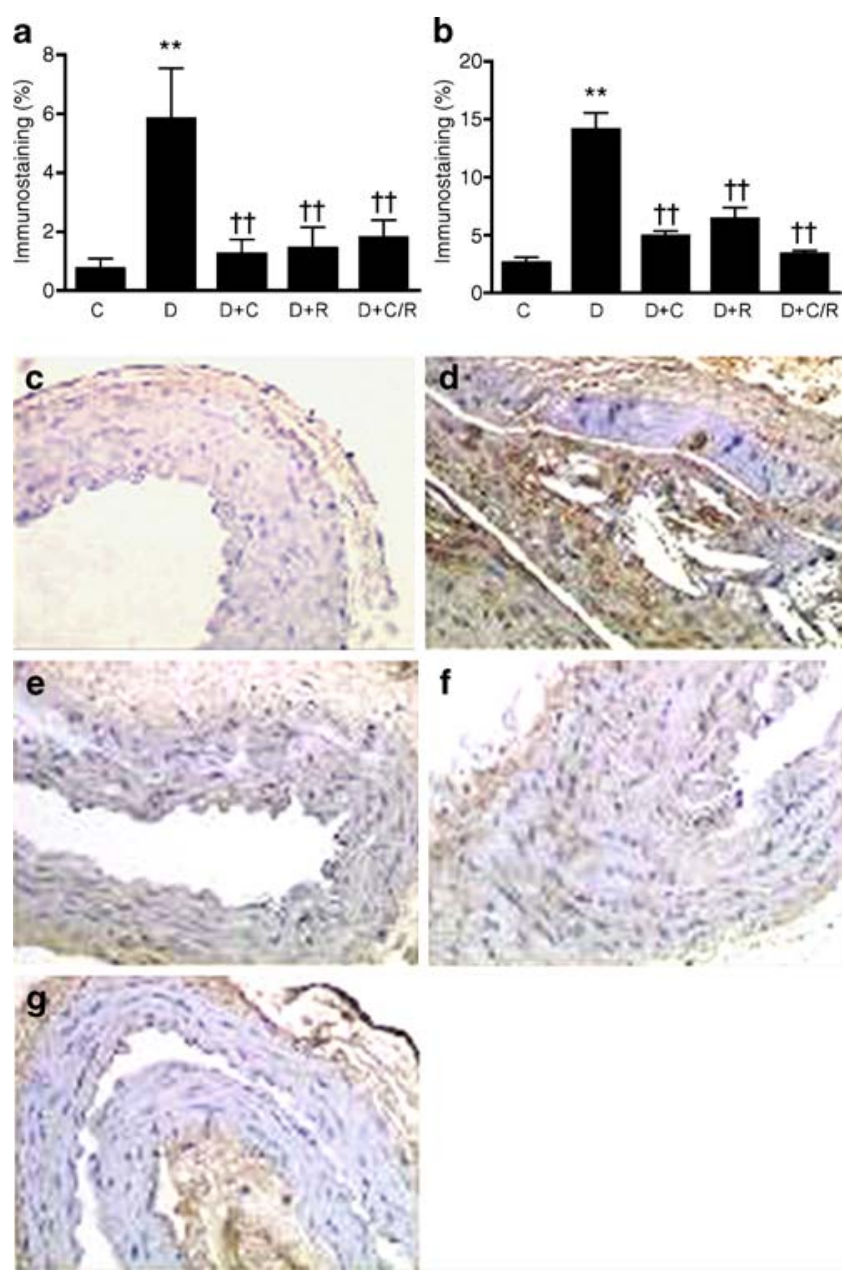

Fig. 4 Immunostaining of nitrotyrosine in the plaque (a) and vessel wall (b). Representative images of vessels of control (c), diabetic (d), diabetic + candesartan (e), diabetic + rosuvastatin (f) and diabetic + candesartan/rosuvastatin (g) mice. Data are expressed as mean \pm SEM. ${ }^{* *} p<0.01$ vs control; ${ }^{\dagger \dagger} p<0.01$ vs diabetic. Magnification $\times 430$. C, control; $\mathrm{D}$, diabetic; $\mathrm{D}+\mathrm{C}$, diabetic + candesartan; $\mathrm{D}+\mathrm{R}$, diabetic + rosuvastatin; $\mathrm{D}+\mathrm{C} / \mathrm{R}$, diabetic + candesartan + rosuvastatin increased in aortas of diabetic Apoe $e^{-/-}$mice and was mildly reduced in the plaques by all three treatment groups, although changes were not statistically significant (ESM Fig. 2). In the adjacent vessel wall, RAC-1 was significantly upregulated in association with diabetes $(p<0.01)$ and was attenuated to control levels by rosuvastatin $(p<$ $0.01)$ and dual therapy $(p<0.01)$.

Smooth muscle cell infiltration Diabetes was associated with an approximately sixfold increase in smooth muscle cell accumulation within plaques $(p<0.05)$ as assessed by $\alpha$-smooth muscle cell actin abundance (ESM Fig. 3).
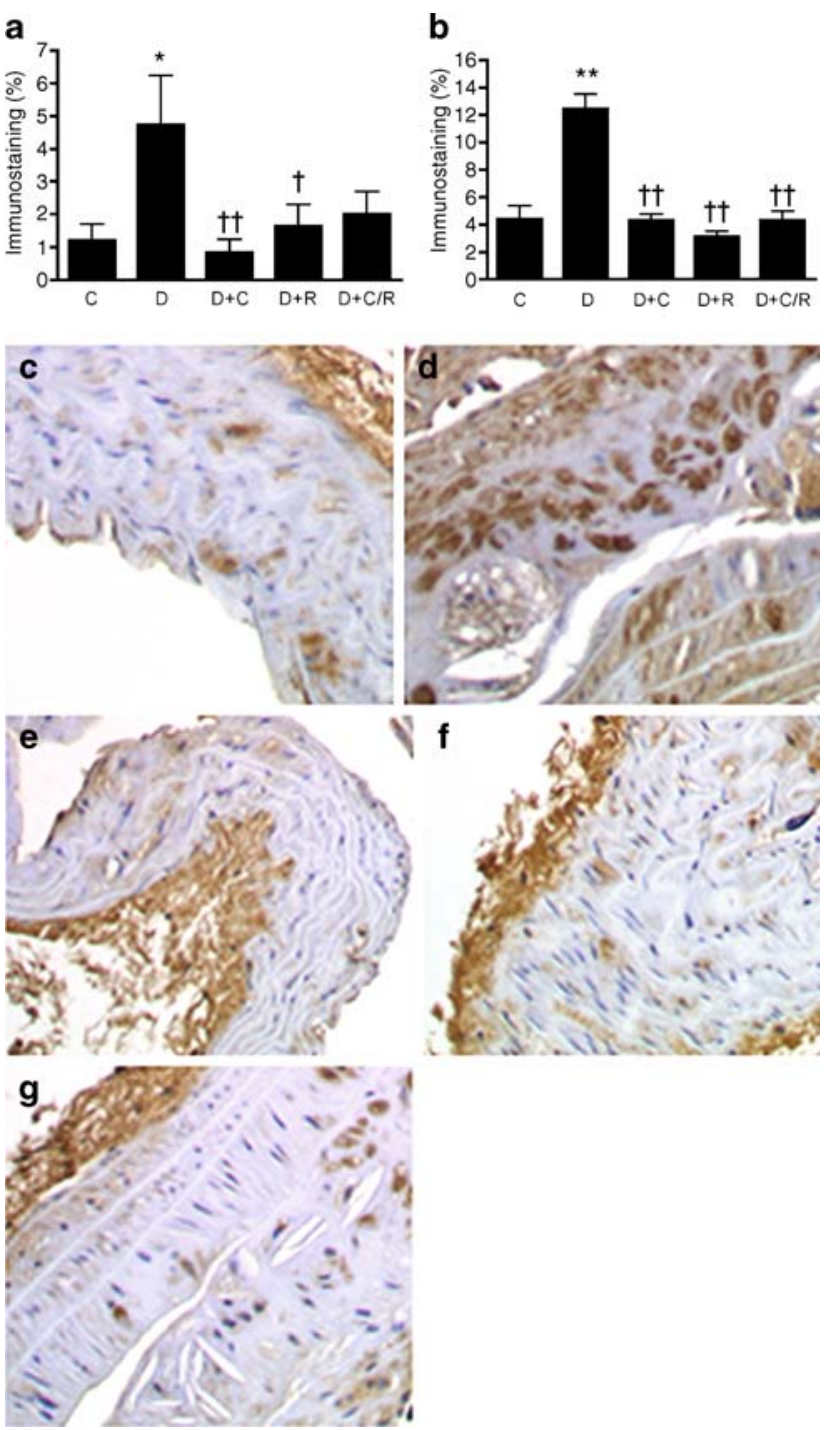

Fig. 5 Immunostaining of the $\mathrm{NAD}(\mathrm{P}) \mathrm{H}$ oxidase subunit $\mathrm{p} 47 \mathrm{phox}$ in the plaque (a) and vessel wall (b). Representative images of vessels of control (c), diabetic (d), diabetic + candesartan (e), diabetic + rosuvastatin (f) and diabetic + candesartan/rosuvastatin (g) mice. Data are expressed as mean \pm SEM. ${ }^{*} p<0.05,{ }^{* *} p<0.01$ vs control; ${ }^{\dagger} p<$ $0.05,{ }^{\dagger} p<0.01$ vs diabetic. Magnification $\times 430$. C, control; D, diabetic; $\mathrm{D}+\mathrm{C}$, diabetic + candesartan; $\mathrm{D}+\mathrm{R}$, diabetic + rosuvastatin; $\mathrm{D}+\mathrm{C} / \mathrm{R}$, diabetic + candesartan + rosuvastatin 
Rosuvastatin $(p<0.01)$, candesartan $(p<0.05)$ and dual therapy $(p<0.01)$ were all effective at attenuating smooth muscle cell infiltration. With respect to the vessel wall, the induction of diabetes was associated with an increase in $\alpha$ smooth muscle actin staining, which was attenuated by rosuvastatin $(p<0.05)$ and dual therapy $(p<0.05)$; candesartan also had an effect, albeit more modest, on this parameter.

Markers of fibrosis The plaques of diabetic mice tended to exhibit increased abundance of collagen I compared with control mice; however, this did not reach significance (Table 2). Candesartan was most effective at attenuating collagen I abundance in the plaques $(p<0.05)$. In the adjacent vessel wall, there was a threefold increase in collagen I abundance in diabetic mice. Interestingly, rosuvastatin was the most effective treatment for attenuating collagen I abundance in the vessel wall adjacent to the plaque $(p<0.05)$.

A similar pattern was seen with collagen IV in plaques, with diabetic mice displaying increased collagen IV compared with control mice, although this did not reach statistical significance (Table 2). None of the treatments significantly altered collagen IV abundance in the plaques. The diabetes-associated upregulation of collagen IV within the vessel wall was more marked $(p<0.01)$. All three treatments were equally effective at attenuating collagen IV production in the vessel wall $(p<0.01)$.

Connective tissue growth factor (CTGF) abundance was markedly increased in plaques of diabetic mice compared with control mice $(p<0.01$; ESM Fig. 4). All three treatments were effective at attenuating CTGF expression to control levels (rosuvastatin, $p<0.01$ ) or lower (candesartan and combination), with the combination therapy being the most effective. In the vascular wall, the level of CTGF was increased in diabetes and tended to be reduced by rosuvastatin and in particular by combination treatment, but changes were not statistically significant.

\section{Discussion}

Diabetic animals demonstrated significantly increased plaque areas after 20 weeks of untreated diabetes. Plaque area in the diabetic $A p o e^{-/-}$mouse was significantly reduced by treatment with the HMG-CoA reductase inhibitor rosuvastatin, the AT1 receptor blocker candesartan and by a combination of both drugs. The anti-atherosclerotic effect conferred by rosuvastatin was independent of effects on lipids and was comparable to the anti-atherosclerotic effect conferred by candesartan. The combination treatment was also effective at decreasing plaque area, but was not superior to candesartan or rosuvastatin therapy alone. The anti-atherosclerotic effects conferred by the three treatment regimens were independent of effects on plasma lipids or glycaemic control.

Previously we have demonstrated, using the diabetic Apoe $^{-/-}$mouse, that the renin-angiotensin system plays a significant role in diabetes-accelerated atherosclerosis $[2$, 3]. The ACE inhibitor perindopril and the AT1 receptor blocker irbesartan were able to reduce plaque area after 20 weeks of diabetes in this model [2,3]. Moreover, despite similar effects on blood pressure in that study, irbesartan was more effective than amlodipine in reducing plaque area, suggesting that blood pressure-lowering per se did not have a major effect on plaque reduction [3].

This is the first report to demonstrate that, in a model of diabetes-accelerated atherosclerosis, the diabetic $\mathrm{Apoe}^{-/-}$ mouse, a statin reduces plaque area independently of its effects on lipids, suggesting that these vasculoprotective effects are related to the pleiotropic effects of rosuvastatin. Furthermore, it should be noted that rosuvastatin attenuated many pro-atherosclerotic pathways, similar to effects seen with the AT1 receptor blocker, a treatment known to have anti-atherosclerotic effects in this model by directly interfering with the local renin-angiotensin system in the vascular wall.

There are several studies in the clinical and pre-clinical setting investigating the effect of an ARB and a statin, although none in the context of diabetes. Chen et al treated Apoe $^{-/}$mice fed a high-cholesterol diet with candesartan and rosuvastatin for 12 weeks and demonstrated that simultaneous administration of these agents reduced atherosclerosis to a greater extent than candesartan or rosuvastatin alone [36]. This was associated with a reduction in CD40 and matrix metalloproteases. Similarly, in another model of high-fat-induced atherosclerosis, treatment with valsartan and fluvastatin for 10 weeks decreased atherosclerotic lesions in $A p o e^{-/-}$mice fed a high-cholesterol diet [37]. Changes in oxidative stress and inflammatory parameters were also observed. Administration of olmesartan and pravastatin together to Watanabe heritable hyperlipidaemic rabbits resulted in a greater antiatherogenic effect than monotherapy [38]. In contrast, Apoe ${ }^{-/-}$mice fed a high-fat diet for 12 weeks demonstrated that combination therapy with telmisartan and atorvastatin was not superior to telmisartan alone [39]. However, an additive effect was seen with respect to plasma inflammatory markers such as IL- 10 .

A number of small clinical studies have also demonstrated varying effects with combination therapies. Hussein et al demonstrated an additive antioxidant effect when valsartan was co-administered for 2 months to seven hypercholesterolaemic, hypertensive patients taking fluvastatin [40]. The Endothelial Protection, AT1 Blockade and 
Cholesterol-Dependent Oxidative Stress (EPAS) Trial demonstrated that treatment with pravastatin $(40 \mathrm{mg} /$ day $)$, in 60 patients with stable coronary artery disease prior to elective coronary artery bypass grafting, was associated with an increase in the anti-atherosclerotic endothelial expression quotient $\mathrm{Q}$, including mRNA expression (endothelial nitric oxide synthase and CNP divided by lectin-like oxidised LDL receptor-1, and gp91phox in left internal mammary arteries) [41]. Treatment with irbesartan $(150 \mathrm{mg} /$ day $)$ had no significant effect. However, when combined with pravastatin, it further increased $\ln Q$, but a putative interaction of both therapies on $\ln Q$ was not significant [41]. Finally, the Simvastatin/Enalapril Coronary Atherosclerosis Trial (SCAT) examined the effect of simvastatin treatment in 460 normocholesterolaemic patients [42]. This study demonstrated beneficial effects on quantitative coronary angiographic measurements with simvastatin treatment, an effect not seen with enalapril. Moreover, there was no additive effect of these drugs on the parameters measured. However, it should be noted that in this study interruption of the renin-angiotensin system was with an ACE inhibitor, rather than with an ARB. While these studies show varying effects of combined statin and ARB therapy on cardiovascular disease, none of these studies was carried out in the setting of diabetes.

In the current study, there appeared to be differences in the mechanisms leading to the anti-atherosclerotic effects observed with each drug. Whereas candesartan was effective at reducing inflammation as evidenced by a decrease in macrophage infiltration and MCP-1 levels in the plaque, rosuvastatin did not have such a marked effect on these parameters. Previous studies have shown reduced inflammation in plaques from people with and without type 2 diabetes in response to various statins [17, 43].

Both drugs were effective in reducing AGE accumulation and RAGE accumulation in the plaque. Although such an effect on the AGE/RAGE axis has previously been observed in cell culture experiments [44] and postulated to be important in type 2 diabetic patients for plaque stabilisation [43], this is the first study to demonstrate effects of a statin on the AGE/RAGE pathway in diabetesaccelerated atherosclerosis. Indeed, our study suggests that statins may reduce AGE accumulation and thereby reduce activation of $\mathrm{NAD}(\mathrm{P}) \mathrm{H}$ oxidase, thus linking oxidant stress to altered gene expression via RAGE, as previously suggested in vitro and in gp91phox-deficient mice [45]. Furthermore, this is the first report to describe superior effects on the AGE/RAGE pathway, particularly in the vascular wall, by a combination regimen consisting of candesartan and rosuvastatin.

Both therapies alone and in combination were effective at reducing the abundance of the various $\mathrm{NAD}(\mathrm{P}) \mathrm{H}$ oxidase subunits in the plaque and vessel wall. It is well established that angiotensin II can increase superoxide production via $\mathrm{NAD}(\mathrm{P}) \mathrm{H}$ oxidase-dependent pathways and has been shown to modulate $\mathrm{NAD}(\mathrm{P}) \mathrm{H}$ oxidase subunit abundance [46]. Indeed, AT1 blockade has been associated with a reduction in superoxide production [47]. Withdrawal of statins has also been shown to increase superoxide production in vessels of mice [48]. This effect was absent in gp91phox (also known as $C y b b)^{-1-}$ mice. Furthermore, withdrawal of statins from human umbilical vein endothelial cells resulted in translocation of the NAD(P)H oxidase subunit, RAC-1, to the membrane and an increase in NAD (P)H-induced lucigenin chemiluminescence. It appears that both drugs, candesartan and rosuvastatin, act on this pathway in a similar manner, modulating $\mathrm{NAD}(\mathrm{P}) \mathrm{H}$ oxidase subunit abundance. Furthermore, interruption of the NAD $(\mathrm{P}) \mathrm{H}$ oxidase pathway results in reduced superoxide production, which could explain the reduction in nitrotyrosine present in the plaques and adjacent vessel wall, as seen with both therapies alone and in combination. Moreover, a reduction in ROS may, in part, be responsible for the attenuation in AGE accumulation as well as for reduced abundance of the pro-inflammatory RAGE, as was seen with both therapies since AGE such as CML are indeed glycoxidation products [49].

Interestingly, while the treatments were effective at attenuating plaque deposition in the aortic arch and abdominal segment, there was no significant effect in the thoracic region. This may partially be due to the lower deposition of plaque within this region. Moreover, it may be due to the specific nature of the effect of the treatment on the vessel wall. For example, treatment of diabetic Apoe $^{-/-}$mice with inhibitors of AGE formation resulted in an attenuation of atherosclerosis in the thoracic and abdominal regions with no effect seen in the aortic arch, whereas treatment with the peroxisome proliferator-activated receptor- $\gamma$ agonist rosiglitazone, postulated to have direct effects on the vessel wall, was associated with most marked effects within the aortic arch [4, 31, 32].

Diabetes is associated with an upregulation of the reninangiotensin system $[2,3]$ in particular within the vascular wall. In addition, at sites of diabetes-related end-organ injury there is increased oxidative stress and inflammation. Thus, it was anticipated that therapies targeting these pathways would confer superior vascular protection in the setting of diabetes. However, in the current study, the combination treatment with candesartan and rosuvastatin did not confer additional anti-atherosclerotic effects as assessed by aortic plaque area. There are several potential explanations for these findings. It is possible that the antiatherosclerotic effect achieved with candesartan was already maximal, as the plaque areas in the candesartan treated diabetic mice were similar to those observed in nondiabetic Apoe $^{-/-}$mice. 
However, the combination treatment clearly exerted superior effects on key mediators of atherosclerosis such as AGE accumulation and RAGE accumulation, as well as on macrophage accumulation and abundance of the chemokine MCP-1, and on the profibrotic cytokine CTGF. Although this superior effect on key mediators of atherosclerosis did not translate into a further reduction of plaque area in the time course of this study, it may be speculated that the more effective suppression of the AGE/RAGE pathway and oxidative stress parameters by the combination regimen might lead to superior vasculoprotection including improved plaque composition and stability, if studied for longer.

In conclusion, the HMG-CoA reductase inhibitor rosuvastatin and the ARB candesartan, both well-known treatments used in diabetic patients at high risk of vascular disease, conferred similar anti-atherosclerotic effects in a model of diabetes-accelerated atherosclerosis, the diabetic Apoe ${ }^{-/-}$mouse, via effects on the AGE/RAGE axis and oxidative stress. These direct pleiotropic vasculoprotective effects were independent of effects on metabolic control and lipids. The anti-atherosclerotic effect conferred by rosuvastatin was comparable to that observed by treatment with the AT1 receptor blocker candesartan, a drug type known to be anti-atherosclerotic in this model, via a mechanism that interrupts the local vascular renin-angiotensin system. The combination of candesartan and rosuvastatin conferred superior effects on the AGE/RAGE pathway, inflammation and oxidative stress.

These studies provide strong evidence for direct beneficial vascular effects of statins and ARBs in diabetesassociated atherosclerosis. Moreover, the vasculoprotection observed is independent of effects on metabolic control, lipids and blood pressure. In particular, the combination treatment of diabetic $A_{p o e^{--}}$mice with the statin rosuvastatin and the ARB candesartan was associated with more effective suppression of AGE accumulation and RAGE accumulation, macrophage accumulation, MCP-1 abundance and oxidative stress parameters, all well-known key mediators in atherosclerosis. Therefore, the combination regimen of both agents has the potential to exert superior long-term vasculoprotection, in particular in the high-risk diabetic patient.

Acknowledgements This work was supported by grants from the Juvenile Diabetes Research Foundation, the National Health and Medical Research Council and National Heart Foundation of Australia. A. C. Calkin is supported by a National Health \& Medical Research Council Postdoctoral Fellowship. K. A. Jandeleit-Dahm is funded by a National Health and Medical Research/ National Heart Foundation Career Development Award.

Duality of interest This study was partially funded by a grant from AstraZeneca.

\section{References}

1. Pyorala K, Uusitupa M, Laakso M et al (1987) Macrovascular complications in relation to hyperinsulinaemia in non-insulindependent diabetes mellitus. Diabetes Metab 13:345-349

2. Candido R, Jandeleit-Dahm KA, Cao Z et al (2002) Prevention of accelerated atherosclerosis by angiotensin-converting enzyme inhibition in diabetic apolipoprotein E-deficient mice. Circulation 106:246-253

3. Candido R, Allen TJ, Lassila M et al (2004) Irbesartan but not amlodipine suppresses diabetes-associated atherosclerosis. Circulation 109:1536-1542

4. Forbes JM, Yee LT, Thallas V et al (2004) Advanced glycation end product interventions reduce diabetes-accelerated atherosclerosis. Diabetes 53:1813-1823

5. Goldberg IJ (2004) Why does diabetes increase atherosclerosis? I don't know! J Clin Invest 114:613-615

6. Collins R, Armitage J, Parish S et al (2003) MRC/BHF Heart Protection Study of cholesterol-lowering with simvastatin in 5963 people with diabetes: a randomised placebo-controlled trial. Lancet 361:2005-2016

7. Pyorala K, Pedersen TR, Kjekshus J et al (1997) Cholesterol lowering with simvastatin improves prognosis of diabetic patients with coronary heart disease. A subgroup analysis of the Scandinavian Simvastatin Survival Study (4S). Diabetes Care 20:614-620

8. Sever PS, Dahlof B, Poulter NR et al (2003) Prevention of coronary and stroke events with atorvastatin in hypertensive patients who have average or lower-than-average cholesterol concentrations, in the Anglo-Scandinavian Cardiac Outcomes Trial-Lipid Lowering Arm (ASCOT-LLA): a multicentre randomised controlled trial. Lancet 361:1149-1158

9. Armitage J, Bowman L (2004) Cardiovascular outcomes among participants with diabetes in the recent large statin trials. Curr Opin Lipidol 15:439-446

10. Keech A, Colquhoun D, Best J et al (2003) Secondary prevention of cardiovascular events with long-term pravastatin in patients with diabetes or impaired fasting glucose: results from the LIPID trial. Diabetes Care 26:2713-2721

11. Goldberg RB, Mellies MJ, Sacks F et al (1998) Cardiovascular events and their reduction with pravastatin in diabetic and glucose-intolerant myocardial infarction survivors with average cholesterol levels: subgroup analyses in the cholesterol and recurrent events (CARE) trial. The Care Investigators. Circulation 98:2513-2519

12. Colhoun HM, Betteridge DJ, Durrington PN et al (2004) Primary prevention of cardiovascular disease with atorvastatin in type 2 diabetes in the Collaborative Atorvastatin Diabetes Study (CARDS): multicentre randomised placebo-controlled trial. Lancet 364:685-696

13. Dunzendorfer S, Rothbucher D, Schratzberger P et al (1997) Mevalonate-dependent inhibition of transendothelial migration and chemotaxis of human peripheral blood neutrophils by pravastatin. Circ Res 81:963-969

14. Guijarro C, Blanco-Colio LM, Ortego M et al (1998) 3-Hydroxy3-methylglutaryl coenzyme a reductase and isoprenylation inhibitors induce apoptosis of vascular smooth muscle cells in culture. Circ Res 83:490-500

15. Hidaka Y, Eda T, Yonemoto M et al (1992) Inhibition of cultured vascular smooth muscle cell migration by simvastatin (MK-733). Atherosclerosis 95:87-94

16. Rogler G, Lackner KJ, Schmitz G (1995) Effects of fluvastatin on growth of porcine and human vascular smooth muscle cells in vitro. Am J Cardiol 76:114A-116A

17. Crisby M, Nordin-Fredriksson G, Shah PK et al (2001) Pravastatin treatment increases collagen content and decreases lipid 
content, inflammation, metalloproteinases, and cell death in human carotid plaques: implications for plaque stabilization. Circulation 103:926-933

18. No authors listed (1993) Hypertension in Diabetes Study (HDS): I. Prevalence of hypertension in newly presenting type 2 diabetic patients and the association with risk factors for cardiovascular and diabetic complications. J Hypertens 11:309-317

19. Turner RC, Millns H, Neil HA et al (1998) Risk factors for coronary artery disease in non-insulin dependent diabetes mellitus: United Kingdom Prospective Diabetes Study (UKPDS: 23). BMJ 316:823-828

20. Niklason A, Hedner T, Niskanen L et al (2004) Development of diabetes is retarded by ACE inhibition in hypertensive patients-a subanalysis of the Captopril Prevention Project (CAPPP). J Hypertens 22:645-652

21. Tatti P, Pahor M, Byington RP et al (1998) Outcome results of the Fosinopril Versus Amlodipine Cardiovascular Events Randomized Trial (FACET) in patients with hypertension and NIDDM. Diabetes Care 21:597-603

22. Fox KM (2003) Efficacy of perindopril in reduction of cardiovascular events among patients with stable coronary artery disease: randomised, double-blind, placebo-controlled, multicentre trial (the EUROPA study). Lancet 362:782-788

23. Lindholm LH, Ibsen H, Dahlof B et al (2002) Cardiovascular morbidity and mortality in patients with diabetes in the Losartan Intervention For Endpoint reduction in hypertension study (LIFE): a randomised trial against atenolol. Lancet 359:1004-1010

24. Chen XL, Tummala PE, Olbrych MT et al (1998) Angiotensin II induces monocyte chemoattractant protein-1 gene expression in rat vascular smooth muscle cells. Circ Res 83:952-959

25. Tham DM, Martin-McNulty B, Wang YX et al (2002) Angiotensin II is associated with activation of NF-kappaB-mediated genes and downregulation of PPARs. Physiol Genomics 11:21-30

26. Daemen MJ, Lombardi DM, Bosman FT et al (1991) Angiotensin II induces smooth muscle cell proliferation in the normal and injured rat arterial wall. Circ Res 68:450-456

27. Zahradka P, Werner JP, Buhay S et al (2002) NF-kappaB activation is essential for angiotensin II-dependent proliferation and migration of vascular smooth muscle cells. J Mol Cell Cardiol 34:1609-1621

28. Rajagopalan S, Kurz S, Munzel T et al (1996) Angiotensin IImediated hypertension in the rat increases vascular superoxide production via membrane NADH/NADPH oxidase activation. Contribution to alterations of vasomotor tone. J Clin Invest 97:1916-1923

29. Sato H, Watanabe A, Tanaka T et al (2003) Regulation of the human tumor necrosis factor-alpha promoter by angiotensin II and lipopolysaccharide in cardiac fibroblasts: different cis-acting promoter sequences and transcriptional factors. J Mol Cell Cardiol 35:1197-1205

30. Naftilan AJ, Pratt RE, Dzau VJ (1989) Induction of plateletderived growth factor A-chain and c-myc gene expressions by angiotensin II in cultured rat vascular smooth muscle cells. J Clin Invest 83:1419-1424

31. Calkin AC, Forbes JM, Smith C et al (2005) Rosiglitazone attenuates atherosclerosis in a model of insulin insufficiency independent of its metabolic effects. Arterioscler Thromb Vasc Biol 25:1903-1909

32. Calkin AC, Cooper ME, Jandeleit-Dahm KA et al (2006) Gemfibrozil decreases atherosclerosis in experimental diabetes in association with a reduction in oxidative stress and inflammation. Diabetologia 49:766-774

33. Krege JH, Hodgin JB, Hagaman JR et al (1995) A noninvasive computerized tail-cuff system for measuring blood pressure in mice. Hypertension 25:1111-1115
34. Cefalu WT, Wang ZQ, Bell-Farrow A et al (1994) Glycohemoglobin measured by automated affinity HPLC correlates with both short-term and long-term antecedent glycemia. Clin Chem 40:1317-1321

35. Friedewald WT, Levy RI, Fredrickson DS (1972) Estimation of the concentration of low-density lipoprotein cholesterol in plasma, without use of the preparative ultracentrifuge. Clin Chem 18:499 502

36. Chen J, Li D, Schaefer RF et al (2004) Inhibitory effect of candesartan and rosuvastatin on CD40 and MMPs expression in apo-E knockout mice: novel insights into the role of RAS and dyslipidemia in atherogenesis. J Cardiovasc Pharmacol 44:446452

37. Li Z, Iwai M, Wu L et al (2004) Fluvastatin enhances the inhibitory effects of a selective AT1 receptor blocker, valsartan, on atherosclerosis. Hypertension 44:758-763

38. Kato M, Sada T, Mizuno M et al (2005) Effect of combined treatment with an angiotensin II receptor antagonist and an HMGCoA reductase inhibitor on atherosclerosis in genetically hyperlipidemic rabbits. J Cardiovasc Pharmacol 46:556-562

39. Grothusen C, Bley S, Selle T et al (2005) Combined effects of HMG-CoA-reductase inhibition and renin-angiotensin system blockade on experimental atherosclerosis. Atherosclerosis 182:57-69

40. Hussein O, Shneider J, Rosenblat M et al (2002) Valsartan therapy has additive anti-oxidative effect to that of fluvastatin therapy against low-density lipoprotein oxidation: studies in hypercholesterolemic and hypertensive patients. J Cardiovasc Pharmacol 40:28-34

41. Morawietz H, Erbs S, Holtz J et al (2006) Endothelial protection, AT1 blockade and cholesterol-dependent oxidative stress: the EPAS trial. Circulation 114(1 Suppl):I296-I301

42. Teo KK, Burton JR, Buller CE et al (2000) Long-term effects of cholesterol lowering and angiotensin-converting enzyme inhibition on coronary atherosclerosis: The Simvastatin/Enalapril Coronary Atherosclerosis Trial (SCAT). Circulation 102:17481754

43. Cuccurullo C, Iezzi A, Fazia ML et al (2006) Suppression of RAGE as a basis of simvastatin-dependent plaque stabilization in type 2 diabetes. Arterioscler Thromb Vasc Biol 26:2716-2723

44. Fujita M, Okuda H, Tsukamoto O et al (2006) Blockade of angiotensin II receptors reduces the expression of receptors for advanced glycation end products in human endothelial cells. Arterioscler Thromb Vasc Biol 26:e138-e142

45. Wautier MP, Chappey O, Corda S et al (2001) Activation of NADPH oxidase by AGE links oxidant stress to altered gene expression via RAGE. Am J Physiol Endocrinol Metab 280: E685-E694

46. Touyz RM, Chen X, Tabet $F$ et al (2002) Expression of a functionally active gp91phox-containing neutrophil-type $\mathrm{NAD}(\mathrm{P}) \mathrm{H}$ oxidase in smooth muscle cells from human resistance arteries: regulation by angiotensin II. Circ Res 90:1205-1213

47. Zhang H, Schmeisser A, Garlichs CD et al (1999) Angiotensin IIinduced superoxide anion generation in human vascular endothelial cells: role of membrane-bound NADH-/NADPH-oxidases. Cardiovasc Res 44:215-222

48. Vecchione C, Brandes RP (2002) Withdrawal of 3-hydroxy-3methylglutaryl coenzyme A reductase inhibitors elicits oxidative stress and induces endothelial dysfunction in mice. Circ Res 91:173-179

49. Fu MX, Wells-Knecht KJ, Blackledge JA et al (1994) Glycation, glycoxidation, and cross-linking of collagen by glucose. Kinetics, mechanisms, and inhibition of late stages of the Maillard reaction. Diabetes 43:676-683 